\title{
Bazedoxifene enhances paclitaxel efficacy to suppress glioblastoma via altering Hippo/YAP pathway
}

\author{
Weiwei $\mathrm{Fu}^{1^{*}}$, Peng Zhao ${ }^{1^{*}}$, Hong $\mathrm{Li}^{1}$, Haiyang Fu${ }^{1}$, Xuejun $\mathrm{Liu}^{2}$, Yingchao Liu ${ }^{3}$, Jie $\mathrm{Wu}^{3}$, Weiwei Fu${ }^{\circledR}$ \\ 1. Department of Pathology, The Affiliated Hospital of Qingdao University, Qingdao, Shandong 266003, P. R. China; \\ 2. Department of Radiology, The Affiliated Hospital of Qingdao University, Qingdao, Shandong 266003, P. R. China \\ 3. Department of Neurosurgery, Provincial Hospital Affiliated to Shandong University, Jinan, Shandong 250006, P. R. China. \\ *Contributed equally \\ $\triangle$ Corresponding author: Weiwei Fu, M.D., Ph. D., Department of Pathology, The Affiliated Hospital of Qingdao University, 16\# Jiangsu Road, Qingdao, \\ Shandong 266003, P.R. China. Tel: +86-17853299767; E-mail: eer-df@163.com. \\ (C) The author(s). This is an open access article distributed under the terms of the Creative Commons Attribution License (https://creativecommons.org/licenses/by/4.0/). \\ See http://ivyspring.com/terms for full terms and conditions.
}

Received: 2019.07.11; Accepted: 2019.11.13; Published: 2020.01.01

\begin{abstract}
Glioblastoma multiform (GBM) is an aggressive type of brain tumor originated from astrocytes. Owing to the limited therapeutic options, intensive efforts are still being made to find novel treatments for GBM. In this study, we first identified that bazedoxifene bore the ability to reduce cell survival and cell invasion of glioblastoma cells. Furthermore, our results also revealed that bazedoxifene combining with paclitaxel had better efficacy to suppress glioblastoma progression by promoting apoptosis and reducing EMT. Combination of bazedoxifene and paclitaxel also accelerated YAP phosphorylation and inactivation. Importantly, preclinical animal model also verified our in vitro findings. Together, our data not only define the underlying mechanism responsible for action of bazedoxifene on glioblastoma cells but also build strong rational to develop bazedoxifene for the treatment of GBM patients.
\end{abstract}

Key words: Bazedoxifene, Paclitaxel, Glioblastoma, YAP

\section{Introduction}

Glioblastoma multiform (GBM) is the most common brain tumor and it occurs in adults with approximate incidence at 3.2/100 000 [12, 18]. The mainstay treatments for glioblastoma are surgery, radiotherapy and chemotherapy [15]. Although these current therapies show promising effectiveness, GBM patients still have poor survival rate with a median survival of only 15 months [16]. Therefore, intensive studies are focused on finding novel targeted therapies for this disease.

Bazedoxifene is a third-generation selective estrogen receptor modulator (SERM) and has approved by the FDA to treat postmenopausal osteoporosis [10]. In addition, bazedoxifene is also being tested for possible treatment of breast cancer, medulloblastoma and pancreatic cancer $[3,6,19,23]$. These studies have demonstrated that bazedoxifene acts as GP130 inhibitor by competing with IL-6 or
IL-11 for the interaction of GP130, leading to the deactivation of IL-6/GP130 signaling and delayed cancer progression. Bazedoxifene treatment in medulloblastoma cells suppressed cell growth and impaired glycolysis [3]. In triple negative breast cancer cells, bazedoxifene also showed an anti-cancer activity by suppressing cell proliferation, cell invasion and tumor growth [19]. However, the anti-cancer activity of bazedoxifene in glioblastoma and whether it can enhance paclitaxel efficacy to suppress glioblastoma cell growth haven't yet been investigated.

Numerous studies have demonstrated that Hippo signaling is involved in cancer development including glioblastoma [27]. YAP is one of essential components in Hippo signaling cascade. MST1/2 (mammalian sterile 20-like kinases 1/2) complexes with Sav1 (salvador family WW domain-containing 
protein 1) to activate LATS1/2 (large tumor suppressor kinases 1/2), leading to the phosphorylation and degradation of YAP $[26,28]$. While the dephosphorylated YAP works together with many transcription factors to control gene expression such as CTGF and Cyr61 [2]. Amplification of YAP has been reported in several human cancers [25]. YAP overexpression could promote epithelialmesenchymal transition (EMT) in MCF10A cells and promoted tumor growth of liver cancer [14, 24]. All these findings have established the important role of the Hippo pathway in cancer development. Although the significance of YAP has been documented in glioblastoma, small molecules targeting YAP are still intensively being tested.

In this study, we found that bazedoxifene alone or in combination with paclitaxel could suppress cell survival and cell invasion of glioblastoma cells. In vivo mouse model also confirmed that combinational treatment using bazedoxifene and paclitaxel further suppressed glioblastoma tumor growth compared with single treatment. Our data provided rational to develop bazedoxifene as another therapeutic option for glioblastoma, either alone or in combination with other drugs.

\section{Materials and Methods}

\section{Cell culture}

LN229, D456, OMRP, LN18, 1016, U251MG and astrocyte cell lines were purchased from ATCC. Cells were cultured in Dulbecco's modified Eagle's medium (DMEM) (Corning, 10-013-CV) supplemented with $10 \%$ Fetal Bovine Serum (Gibco, 10437028) and 1\% Antibiotic-Antimycotic Solution (Corning, 30-004-CI). Bazedoxifene acetate and paclitaxel were purchased from Acesys Pharmatech (USA) and dissolved in DMSO at the concentration of $20 \mathrm{mM}$ and $5 \mathrm{mg} / \mathrm{ml}$ respectively. ER $\alpha$, Cyclin D, Bcl-2, p-p70S6K, Vimentin, E-cadherin, p-YAP (S127), CTGF, Cyr61, sail, MMP9 and Actin antibodies were purchased from Cell Signaling Technology (Beverly, Massachusetts, USA).

\section{Cell viability assay}

Cell viability was measured using 3-(4, 5-dimethylthiazolyl)-2 and 5-diphenyltetrazolium bromide (MTT) assay. Glioblastoma cells were seeded in 96-well plates at a density of 5000 cells per well. 24 hours later, cells were treated by bazedoxifene or paclitaxel or combination for another 24 hours. Then, $25 \mu \mathrm{l}$ of MTT (Sigma, St Louis, Missouri, USA) was added to each well and incubated for 2 hours. Relative cell number was measured by the absorbance at 570 nm.

\section{Western blotting}

Cell were lysed by RIPA lysis buffer. Equal amount of protein $(20 \mu \mathrm{g})$ was subjected to $8 \%-12 \%$ SDS-PAGE gel separation and transferred to a polyvinylidene difluoride membrane. After being blocked by $10 \%$ BSA, the membranes were incubated with specific primary antibodies at $4^{\circ} \mathrm{C}$ overnight. Then the membranes were washed by Tris-buffered saline with $0.1 \%$ Tween-20 and incubated with corresponding secondary antibody at room temperature for $1 \mathrm{~h}$. Blots were detected using the ChemiDOC XRS + system (Bio-Rad Laboratories, Hercules, California, USA).

\section{Colony formation assay}

Glioblastoma cells were seeded into 6-cm culture plate at the density of 1000 . Cells were treated by either bazedoxifene or paclitaxel or combination. 2 weeks later, colonies were fixed with cold methanol and stained with $0.1 \%$ crystal violet.

\section{D invasion assay}

Precoat 8-well glass chamber slides with $40 \mu \mathrm{l}$ of matrigel and allow them to solidify in the cell culture incubator. Seed $1 \times 10^{5}$ glioblastoma cells into each well with medium containing 5\% matrigel and $10 \mathrm{ng} / \mathrm{ml}$ EGF. Medium was replenished every 4 days. 2 weeks later, representative images were taken under 200× microscope. Acini-like structures were counted by randomly choosing six fields.

\section{Orthotopic GBM mouse model}

$1 \times 10^{6}$ cells luciferase- cells were directly injected into the brain of six- to 6 -week old nude mice. When tumor size reached to appropriate size, mice were divided into four groups and received the following treatments: 1 ) vehicle; 2 ) bazedoxifene ( $5 \mathrm{mg} / \mathrm{kg}) ; 3$ ) paclitaxel $(10 \mathrm{mg} / \mathrm{kg}) ; 4)$ bazedoxifene plus paclitaxel. Drugs were administered every other day. Tumor size was measured by IVIS. Four weeks later, mice were sacrificed for multiple experiments.

\section{Statistical analysis}

Statistical analysis was carried out using GraphPad Prism 6.0 software (GraphPad Software, San Diego, California, USA). Data was shown as mean \pm SE. Differences were analyzed using Student t-test and significance was set at $\mathrm{P}$ value $<0.05$. Each experiment was repeated at least three times.

\section{Results}

\section{Bazedoxifene exhibited anti-cancer activity on glioblastoma cells}

Bazedoxifene has been approved to suppress bone tumor, breast cancer and pancreatic cancer in 
vitro and in vivo. To test whether bazedoxifene exerted anti-cancer activity on glioblastoma cells, we treated several glioblastoma cell lines (LN229, D456, OMRP, LN18 and 1016) with bazedoxifene for 24 hours. Data showed that bazedoxifene decreased cell viability of these glioblastoma cells in a dose dependent manner (Figure 1A). As control, normal astrocytes failed to respond to bazedoxifene (Figure 1A), indicating bazedoxifene bore an anti-cancer activity on glioblastoma cells without affecting normal cells. At molecular level, bazedoxifene treatment led to downregulation of $\mathrm{ER} \alpha$, Cyclin $\mathrm{D}$, Bcl-2 and inactivation of mTOR signaling monitored by p-P70S6K (Figure B-D) in LN229, D456 and OMRP cells. Consistently, no apparent alteration of ER $\alpha$, Cyclin D, Bcl-2 and mTOR was observed in astrocytes in the presence of bazedoxifene (Figure 1E).
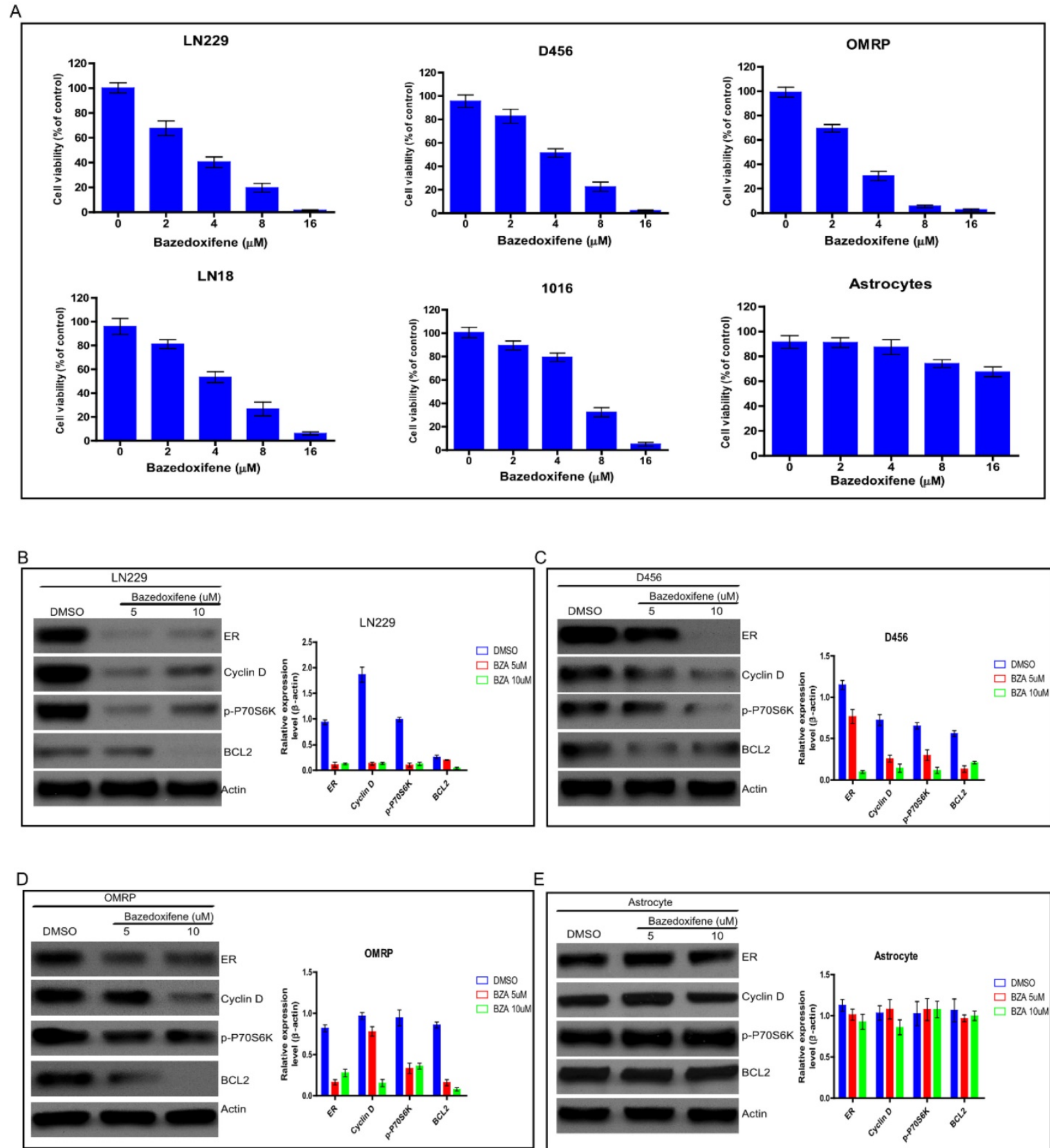

Figure 1. Bazedoxifene exhibited anti-cancer activity on glioblastoma cells. (A). Bazedoxifene dose dependently suppressed cell viability of LN229, D456, OMRP, LN18 and 1016 cells but not normal astrocytes. (B-D). Bazedoxifene treatment reduced the expression levels of ER $\alpha, C y c l i n$ D, Bcl2 and suppressed mTOR activity in LN299 cells (B). D456 cells (C) and OMRP cells (D). ACTIN served as loading control. (E). Bazedoxifene had little effect on the expression levels of ER $\alpha$, Cyclin $\mathrm{D}, \mathrm{Bcl} 2$ in astrocytes. ACTIN was internal control. ${ }^{*} \mathrm{p}<0.1 ; *^{*} \mathrm{p}<0.01 ; *^{* *} \mathrm{p}<0.001$. 


\section{Bazedoxifene combined with paclitaxel synergistically suppressed cell growth of glioblastoma cells}

Paclitaxel is one of potential chemotherapy drugs used for the treatment of glioblastoma patients owing to its anti- microtubule property. Here, we sought to investigate whether combination of paclitaxel with bazedoxifene had better efficacy to suppress glioblastoma cells. Expectedly, bazedoxifene combined with paclitaxel had better ability to decrease cell survival of glioblastoma cells compared to single treated group, assayed by MTT in LN229, D456 and OMRP cells (Figure 2A). As control, astrocytes had no response to these two drugs (Figure 2A). Accordantly, colony formation assay also confirmed that bazedoxifene combined with paclitaxel evidently reduced the number of colonies in LN229, D456, OMRP, LN18 and 1016 cells (Figure 2B).

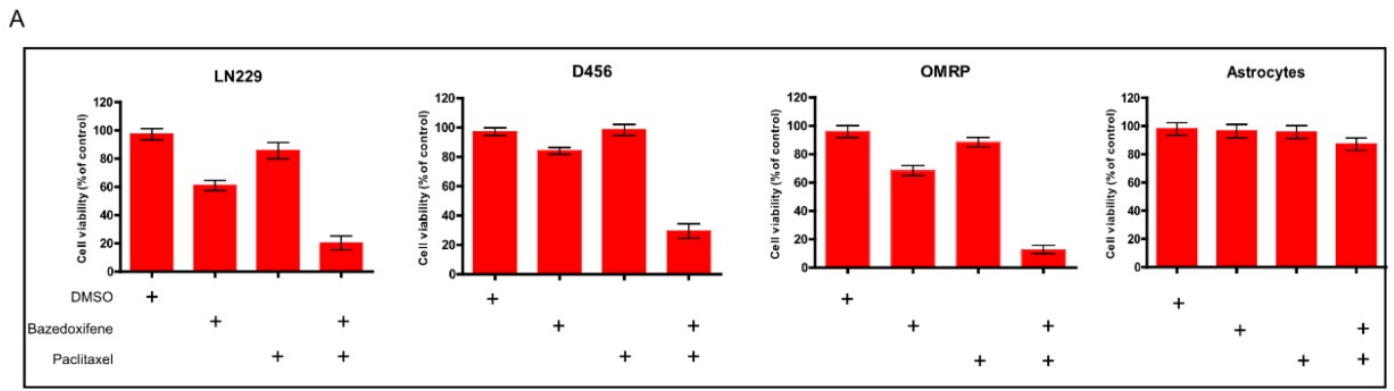

B
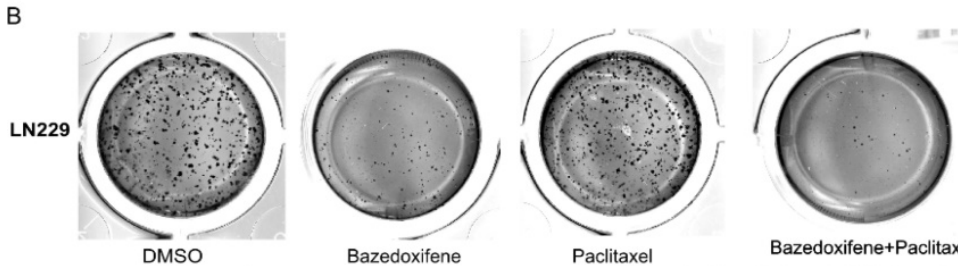

Bazedoxifene+Paclitaxel
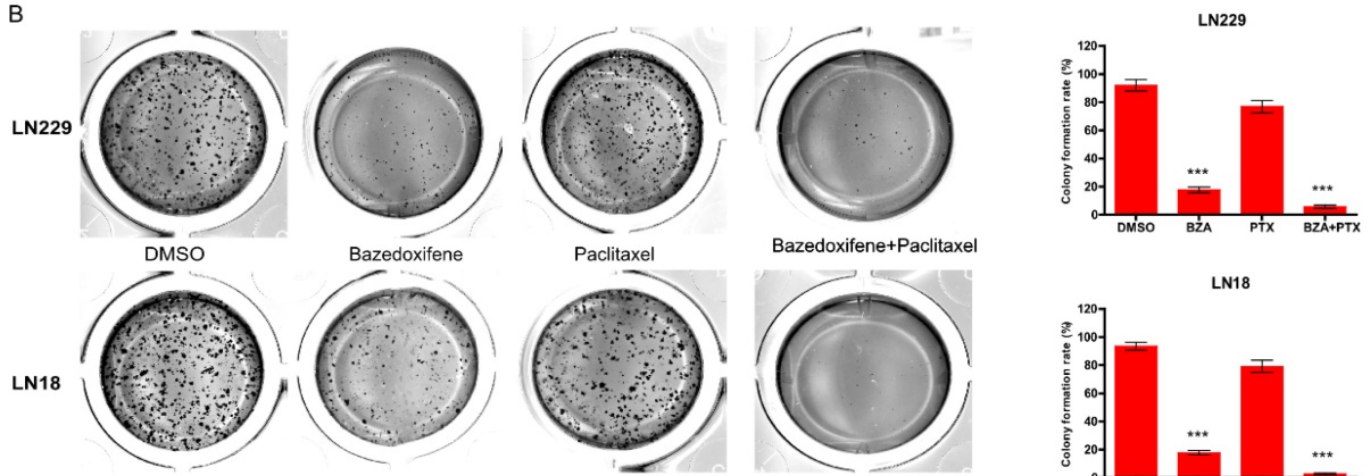

Bazedoxifene

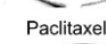

DMSO
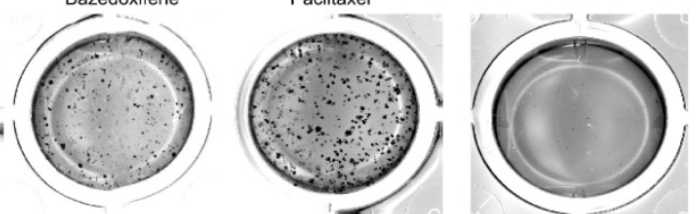

Bazedoxifene+Paclitaxel
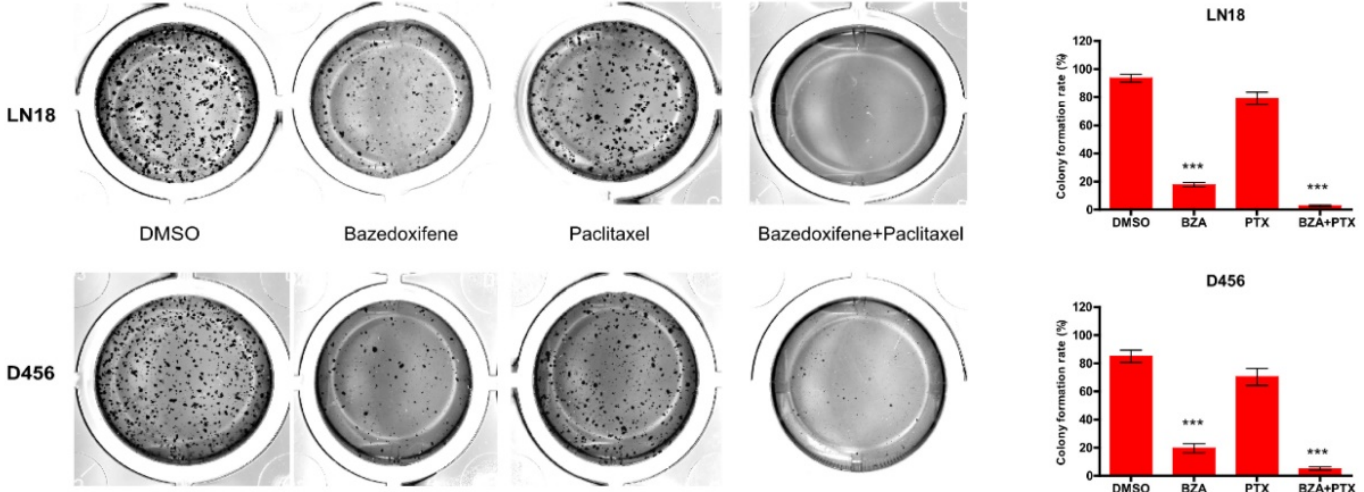

D456
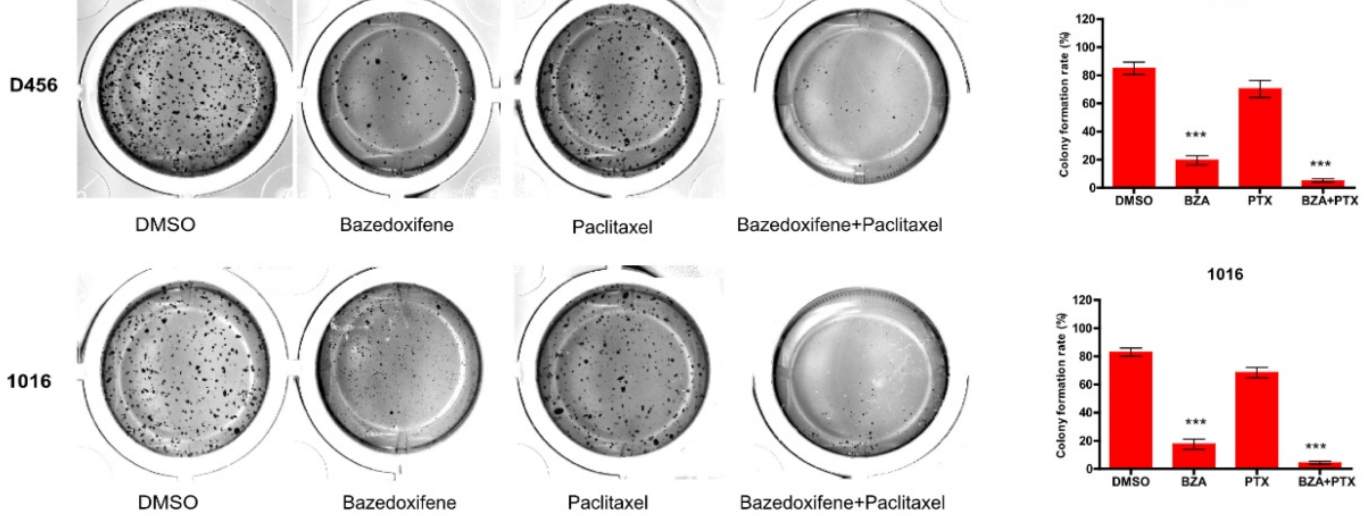

Bazedoxifene+Paclitaxel

1016
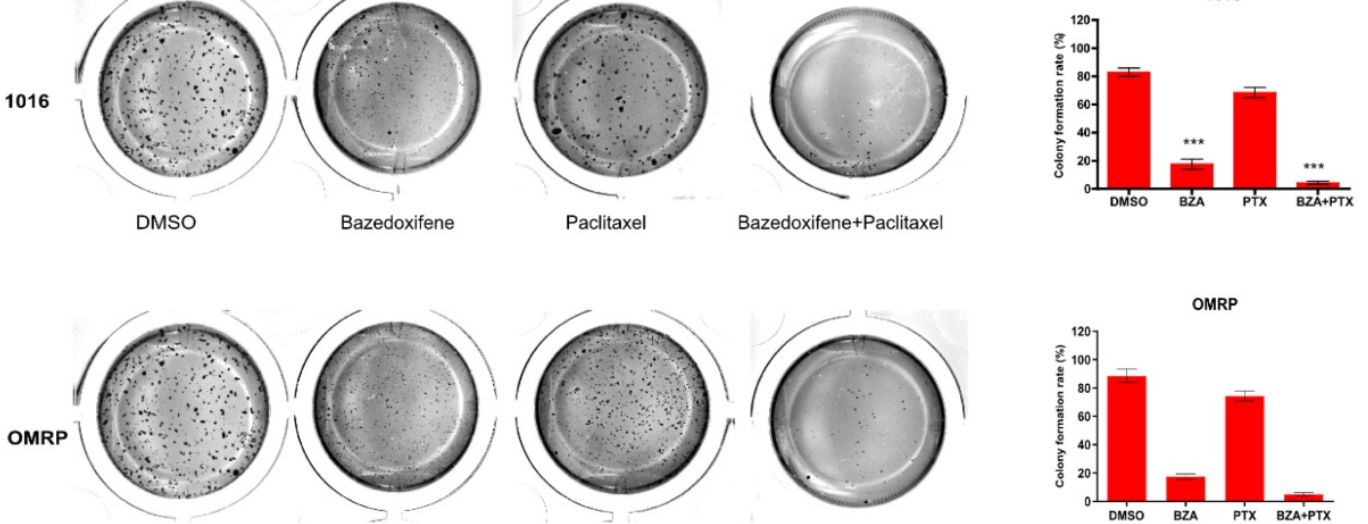

Figure 2. Bazedoxifene combined with paclitaxel synergistically suppressed cell growth of glioblastoma cells. (A). Combination of bazedoxifene $(5 \mu M)$ with paclitaxel $(1 \mu \mathrm{g} / \mathrm{ml})$ suppressed cell viability of LN229, D456, OMRP cells but not astrocytes. (B). Colony formation assay revealed that bazedoxifene (5 $\mu$ M) combined with paclitaxel $(1 \mu \mathrm{g} / \mathrm{ml})$ dramatically reduced cell growth of LN229, D456, OMRP, 1016 and LN18 cells. Left, representative images of colonies with indicated treatments. Right, statistical analyses. ${ }^{*} p<0.1 ;{ }^{*} p<0.01 ; * * * p<0.001$. 


\section{Bazedoxifene combined with paclitaxel triggered a stronger cell apoptosis}

Apoptosis pathway is well linked to cell growth. We next sought to test whether cell apoptosis was involved in bazedoxifen/paclitaxel mediated cell growth suppression of glioblastoma cells. Cleaved caspase- 3 serves as one marker of cell apoptosis. As expected, bazedoxifene alone promoted cleaved caspase-3 production in LN229 cells. However, the combination of bazedoxifene with paclitaxel had

A

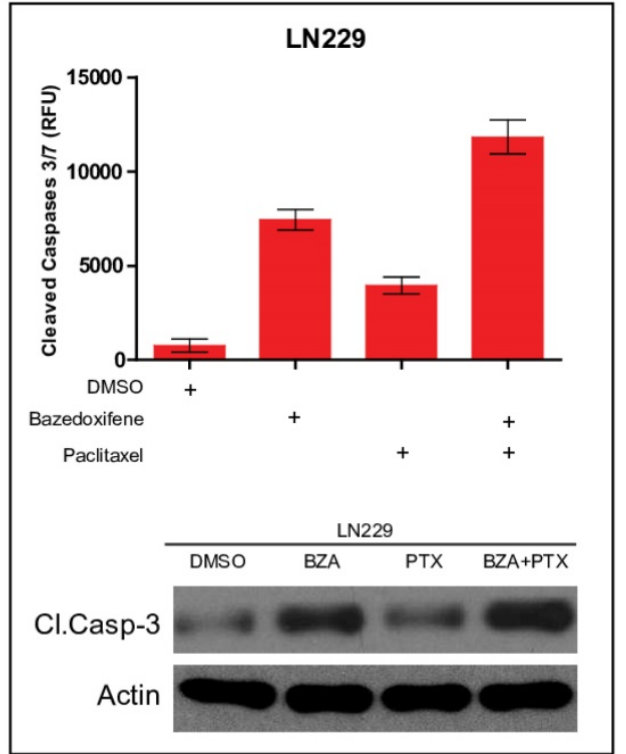

C

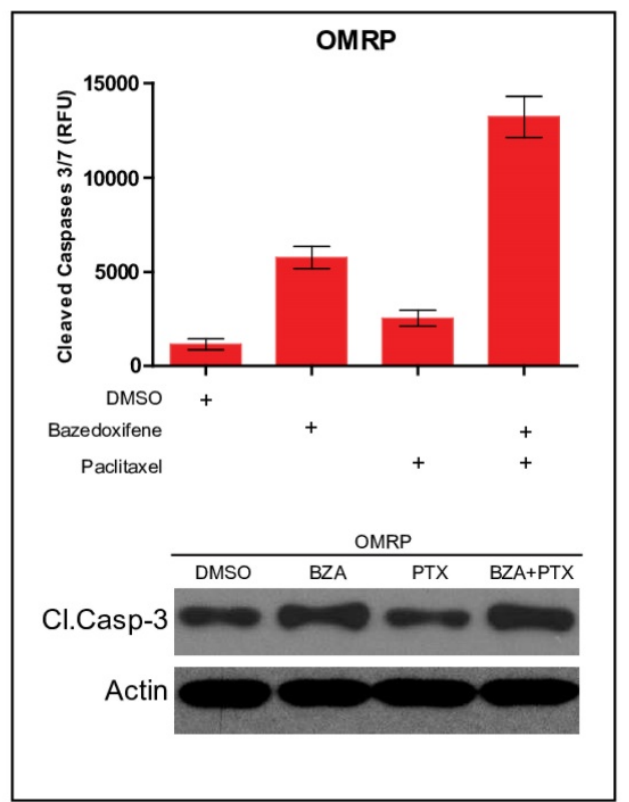

stronger ability to elevate cleaved caspase-3 levels (Figure 3A), suggesting cell apoptosis was involved in bazedoxifen/paclitaxel mediated cell growth suppression of glioblastoma cells. Similar results were obtained when we replaced LN229 cells with D456 (Figure 3B) or OMRP cells (Figure 3C). As control, neither bazedoxifene nor paclitaxel had effect on cleaved caspase-3 levels in normal astrocyte cells (Figure 3D).

B
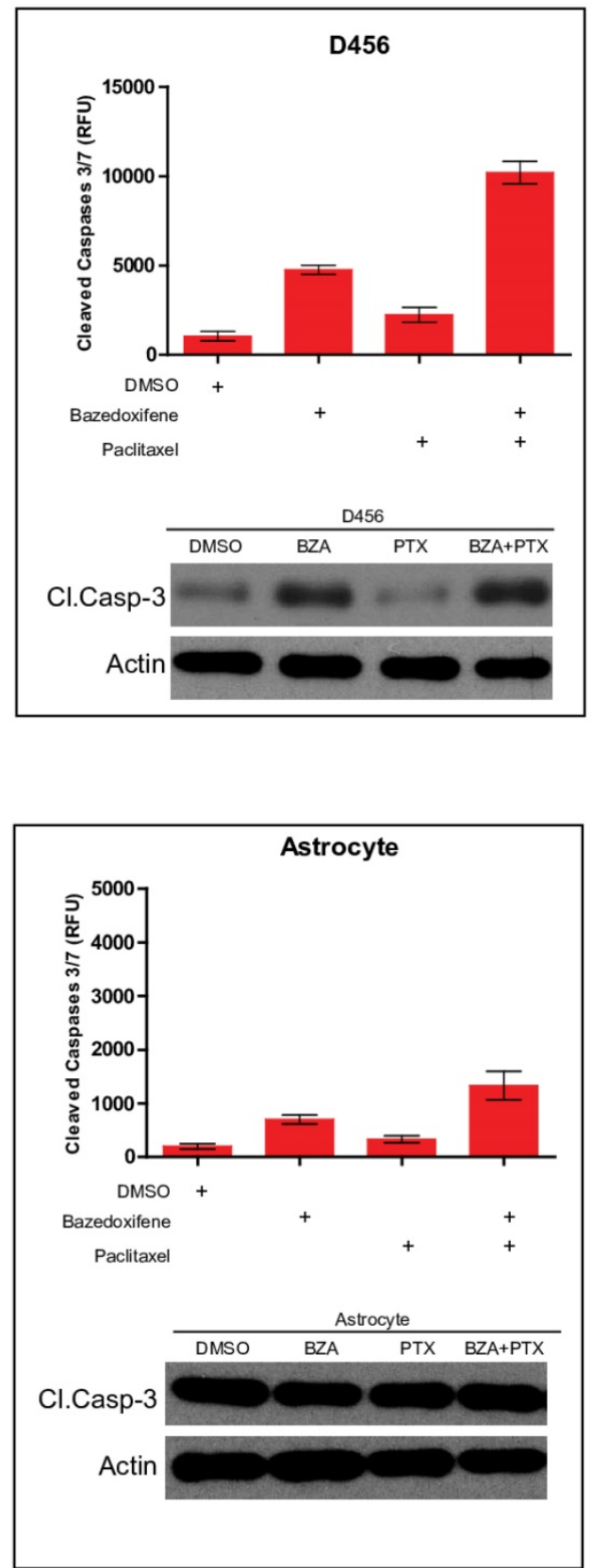

Figure 3. Bazedoxifene combined with paclitaxel triggered a stronger cell apoptosis. Bazedoxifene: $5 \mu$ M; Paclitaxel: $1 \mu g / m l$. (A-C). Western blotting results showed that combination treatment using bazedoxifene (BZA) and paclitaxel (PTX) had stronger ability to promote cleaved caspase-3 production in LN229 (A), D456 (B) and OMRP cells (C). Top, statistical analyses of cleaved caspase-3 blots. Bottom, western blotting detection of cleaved caspase 3 and ACTIN. ACTIN was internal control. (D).Neither bazedoxifene nor paclitaxel had effect on the expression levels of cleaved caspase 3 in astrocytes. ACTIN was used as loading control. ${ }^{*} \mathrm{p}<0.1 ;{ }^{*} \mathrm{p}<0.01$; *** $\mathrm{p}<0.001$ 


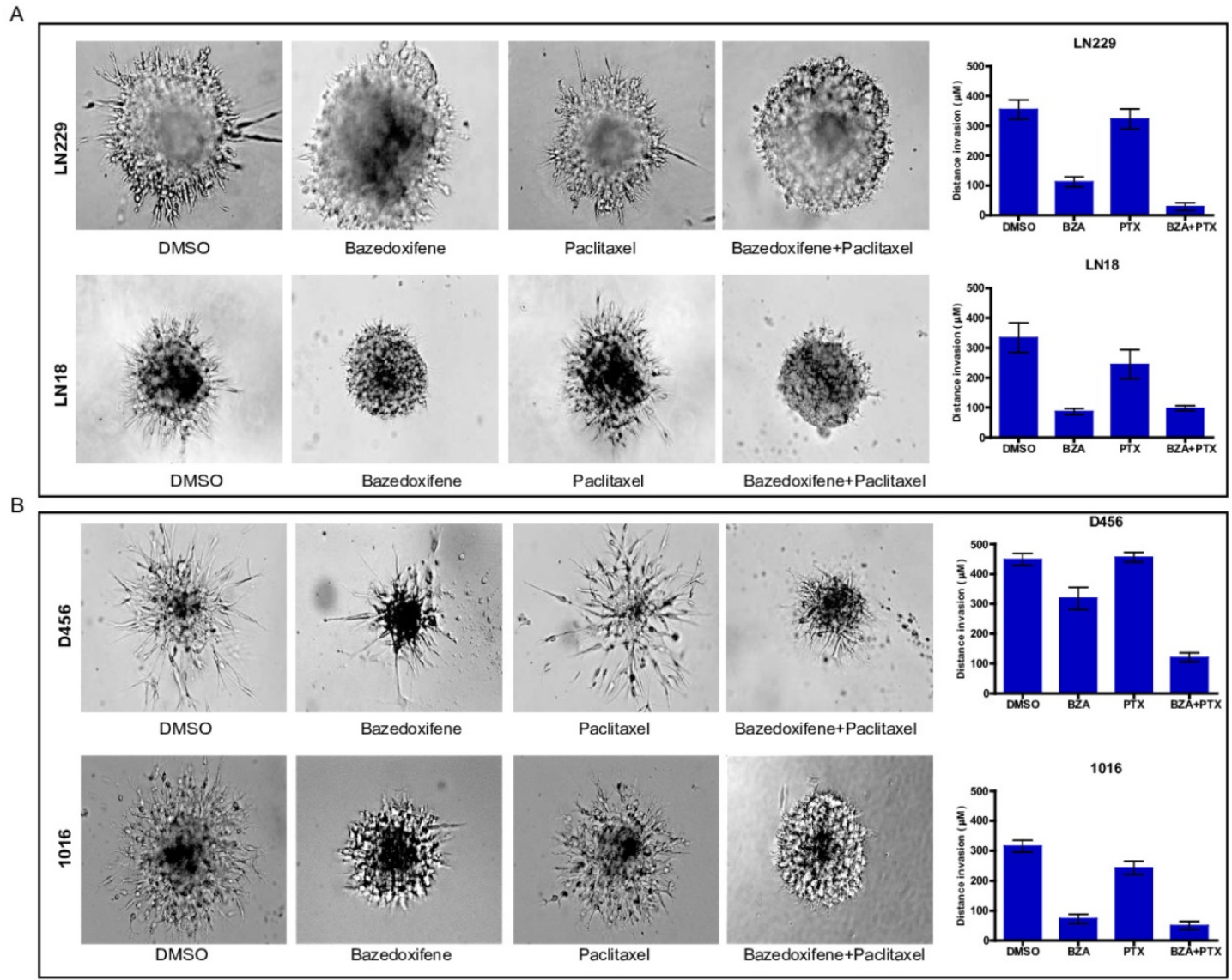

C

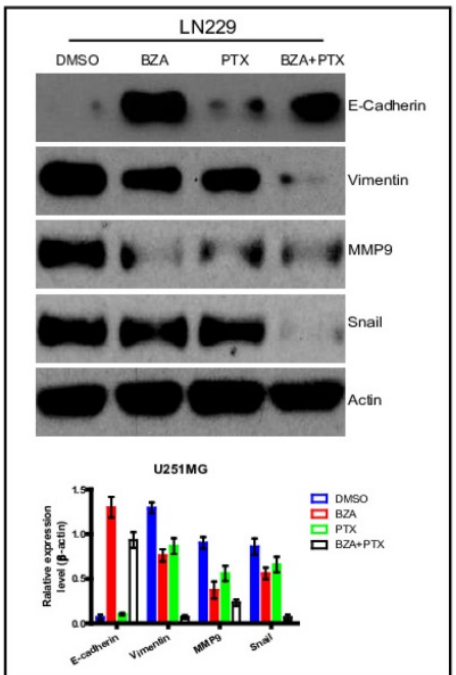

。

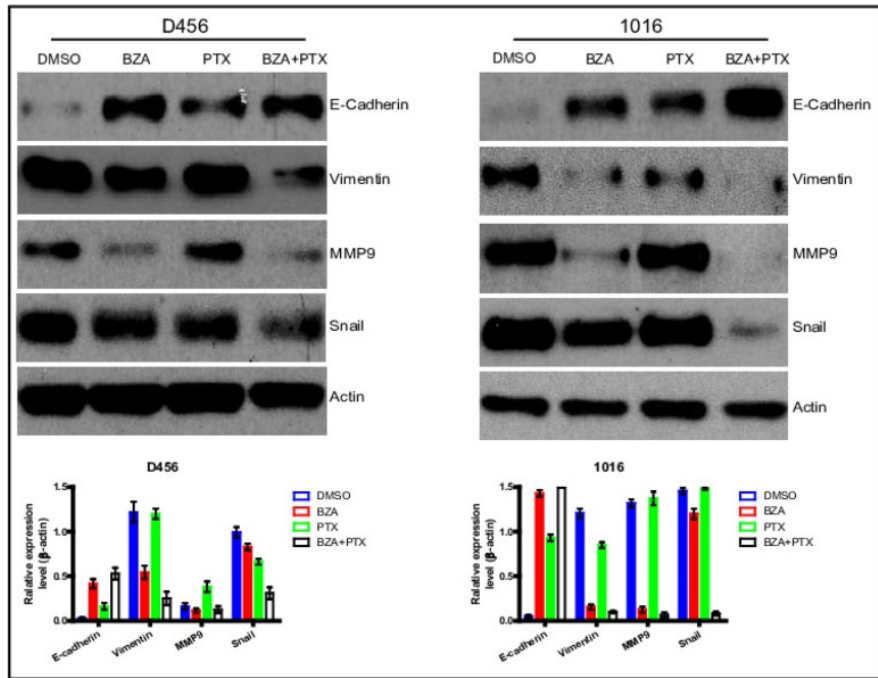

Figure 4. Bazedoxifene combined with paclitaxel further inhibited cell invasion of glioblastoma cells. Bazedoxifene: $5 \mu M$; Paclitaxel: $1 \mu g / m l$. (A). 3-D invasion indicated that bazedoxifene combined with paclitaxel further inhibited cell invasion of LN229 and LN18 cells. Left, representative images. Right, statistical analyses. (B). 3-D invasion showed that bazedoxifene combined with paclitaxel further reduced cell invasion of D456 and 1016 cells. Left, representative images. Right, statistical analyses. (C). Western blotting analyses demonstrated that combination of bazedoxifene with paclitaxel decreased expression levels of vimentin, MMP9 and snail but increased expression levels of E-cadherin in LN299 cells. (D) Real time qPCR analyses demonstrated that combination of bazedoxifene with paclitaxel decreased expression levels of vimentin, MMP9 and snail but increased expression levels of E-cadherin in D456 and 1016 cells. ${ }^{*}<<0.1 ; * * p<0.01 ; * * * p<0.001$

\section{Bazedoxifene combined with paclitaxel further inhibited cell invasion of glioblastoma cells}

Cell invasion is one of essential hallmarks for cancer development. Next, we explored whether bazedoxifene combined with paclitaxel had any effect on cell invasion of glioblastoma cells. To end this, we applied 3-D invasion assay in glioblastoma cells as indicated treatments. Result showed that less acini-like structures were observed in bazedoxifenetreated and paclitaxel treated LN299 cells (Figure 4A). 
Importantly, combination of bazedoxifene with paclitaxel significantly reduced acini-like structures in LN299 cells (Figure 4A, top). We observed similar phenomenon in LN18, D456 and 1016 cells (Figure 4A, bottom \& Figure 4B). Given the fact epithelial mesenchymal transition (EMT) plays essential role in the process of cell invasion, we tested several EMT markers with or without bazedoxifene/paclitaxel treatment. Figure 4C-D displayed that combinational treatment using bazedoxifene and paclitaxel caused considerable upregulation of E-cadherin and downregulation of vimentin, MMP9 and snail in LN229, D456 and 1016 cells, which was consistent to the phenotype we observed.

\section{Bazedoxifene combined with paclitaxel had stronger ability to suppress YAP signaling}

Hippo signaling pathway is involved in numerous biological processes including cell proliferation, organ development [7, 8]. Hippo pathway is also tightly associated with cancer carcinogenesis and development [25]. To test whether alteration of Hippo pathway is responsible for the suppressing effect of bazedoxifene and paclitaxel on glioblastoma cells, we used YAP phosphorylation and its downstream targets (CTGF and Cyr61) as indicators to monitor Hippo pathway. Results showed that bazedoxifene or paclitaxel alone could increase the phosphorylation levels of YAP and decrease the expression levels of CTGF and Cyr61 (Figure 5A-C) in LN229, D456 and OMRP cells. However, combination treatment using bazedoxifene and paclitaxel could further reduce the expression levels of CTGF and Cyr61 (Figure 5A-C). To confirm this finding, we also performed immunofluorescence staining to monitor YAP location. Figure 5D revealed that bazedoxifene plus paclitaxel treatment significantly boosted the translocation of YAP from nucleus to cytosol. Meanwhile, real-time qPCR also verified that bazedoxifene combined with paclitaxel had better capacity to reduce the mRNA levels of CTGF and Cyr61 compared to single treatment (Figure 5E). Together, all these data suggest that activation of Hippo pathway is at least one of mechanisms responsible for bazedoxifene-mediated cell growth suppression in glioblastoma cells.

\section{Bazedoxifene combined with paclitaxel further suppressed glioblastoma tumor growth}

To translate our in vitro findings into pre-clinical animal model, we sought to test the therapeutic efficacy of bazedoxifene either alone or in combination with paclitaxel in orthotopic GBM mouse model. First, we directly implanted 1X106 luciferase U251MG cells into the brain of nude mice. After tumor reached to appropriate size, we randomly divided them into four groups and orally gave the following therapies every other day: 1) vehicle; 2) bazedoxifene (5 $\mathrm{mg} / \mathrm{kg})$; 3) paclitaxel (10 mg/kg); 4) bazedoxifene plus paclitaxel. Tumor growth rate was measured by IVIS. 4 weeks later, mice were sacrificed. Results showed that tumor growth (Figure 6A, a-b) was dramatically suppressed in bazedoxifene/paclitaxel treated group, which was also confirmed by $\mathrm{HE}$ staining (Figure 6A, c). Moreover, the alterations of EMT markers and YAP signaling were consistent to our in vitro results (Figure 6A, d). We also observed an enhanced levels of cleaved caspase 3 , measured by immunofluorescence staining of frozen tissues, in the combinational treatment. We also confirmed these findings in D456 and OMRP based orthotopic GMB model. Results consistently showed that bazedoxifene could enhance paclitaxel efficacy to suppress glioblastoma tumor growth (Figure 6B-C).

\section{Discussion}

Glioblastoma is the most common and lethal brain tumor in adults [22]. It can be classified as primary or secondary malignant brain tumor dependent of cell origination [22]. Primary tumor is the most common form of glioblastoma and is more lethal to people. While, secondary glioblastoma originates from a lower-grade astrocytic tumor and may progress into high-grade tumor over time. Even although current treatments such as surgery, radiation and chemotherapy improve patients' lives a lot $[13,15]$, the poor survival rate puts finding novel therapeutic strategies into the first place. Here, we first found that bazedoxifene could suppress cell growth and cell invasion of glioblastoma. Additionally, bazedoxifene combined with paclitaxel had better efficacy to inhibit glioblastoma progression. Our study finds a novel therapeutic option for the treatment of glioblastoma.

The incidence of glioblastoma has a gender difference: men are approximately 1.5-2 times more likely to have glioblastoma than women [11]. In addition, previous study had demonstrated that glioblastoma cells were easily to develop tumor in male mice than in female mice [9]. However, another study pointed that estrogen could promote glioblastoma tumor growth in orthotopic mouse model [1]. These inconsistent results may tell us that $E R \alpha$ and ER $\beta$ play different roles in glioblastoma carcinogenesis and development. As the third generation selective estrogen receptor modulator, bazedoxifene can act as either estrogen agonist or estrogen antagonist dependent of cancer type. In this study, we found that bazedoxifene treatment evidently reduced the expression of estrogen receptor 
alpha $(E R \alpha)$, implying ER $\alpha$ plays tumor promoting role in glioblastoma development. More efforts should be made to dissect the underlying mechanisms by which ER $\alpha$ promotes glioblastoma progression.
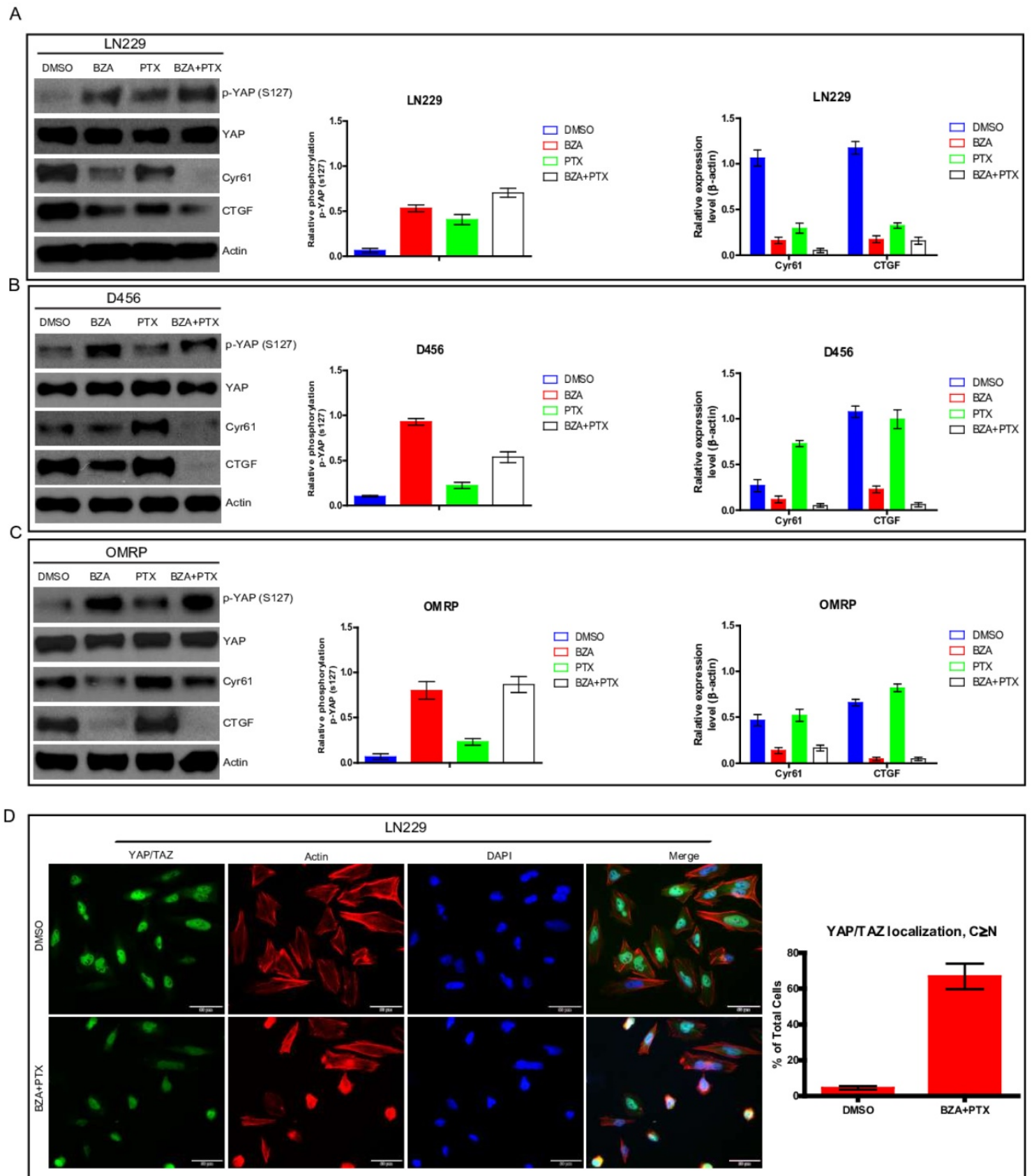

E

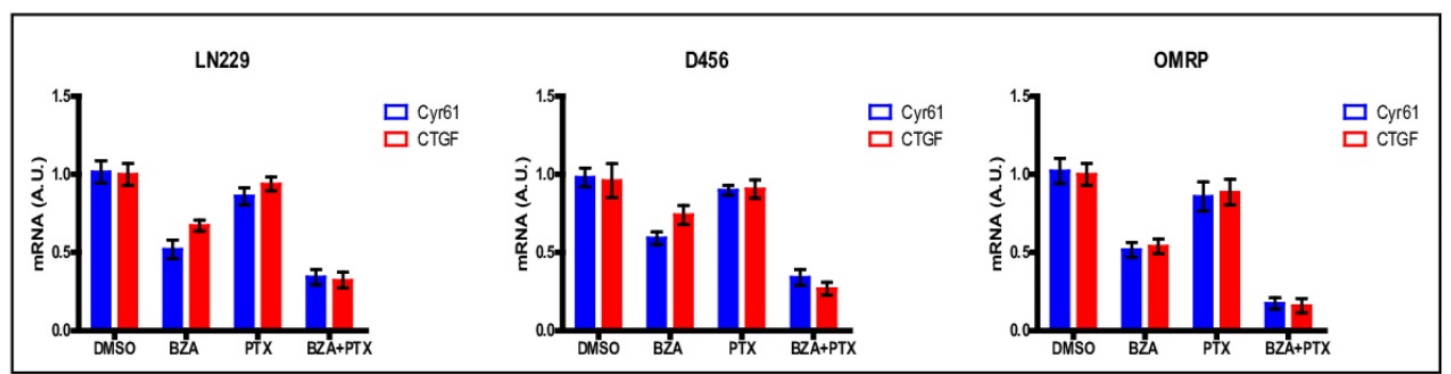

Figure 5. Bazedoxifene combined with paclitaxel had stronger ability to suppress YAP signaling. Bazedoxifene: $5 \mu$ M; Paclitaxel: $1 \mu$ g/ml. (A-C).The phosphorylation levels of YAP (S127) were increased while the expression levels of CTGF and Cyr61 were decreased upon combinational treatment of bazedoxifene (BZA) and paclitaxel (PTX) in LN299 (A), D456 (B) and OMRP cells (C). (D). immunofluorescence staining showed that bazedoxifene combined with paclitaxel led to accumulation of YAP in cytosol. (E). Real time $\mathrm{qPCR}$ showed that the mRNA levels of CTGF and Cyr61 were reduced upon combinational treatment using bazedoxifene and paclitaxel. * ${ }^{*}<0.1 ; * * p<0.01 ; * * p<0.001$ 

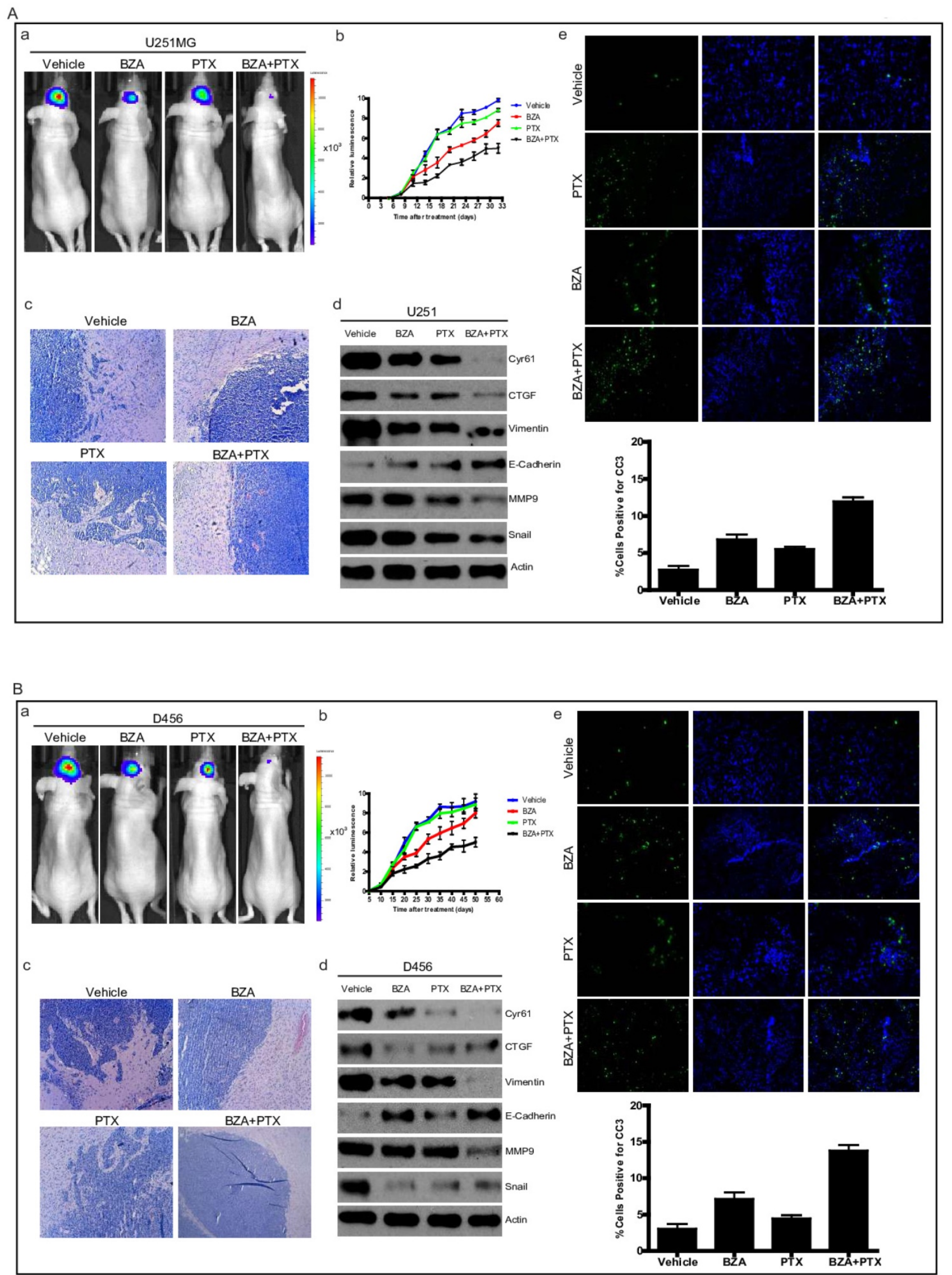


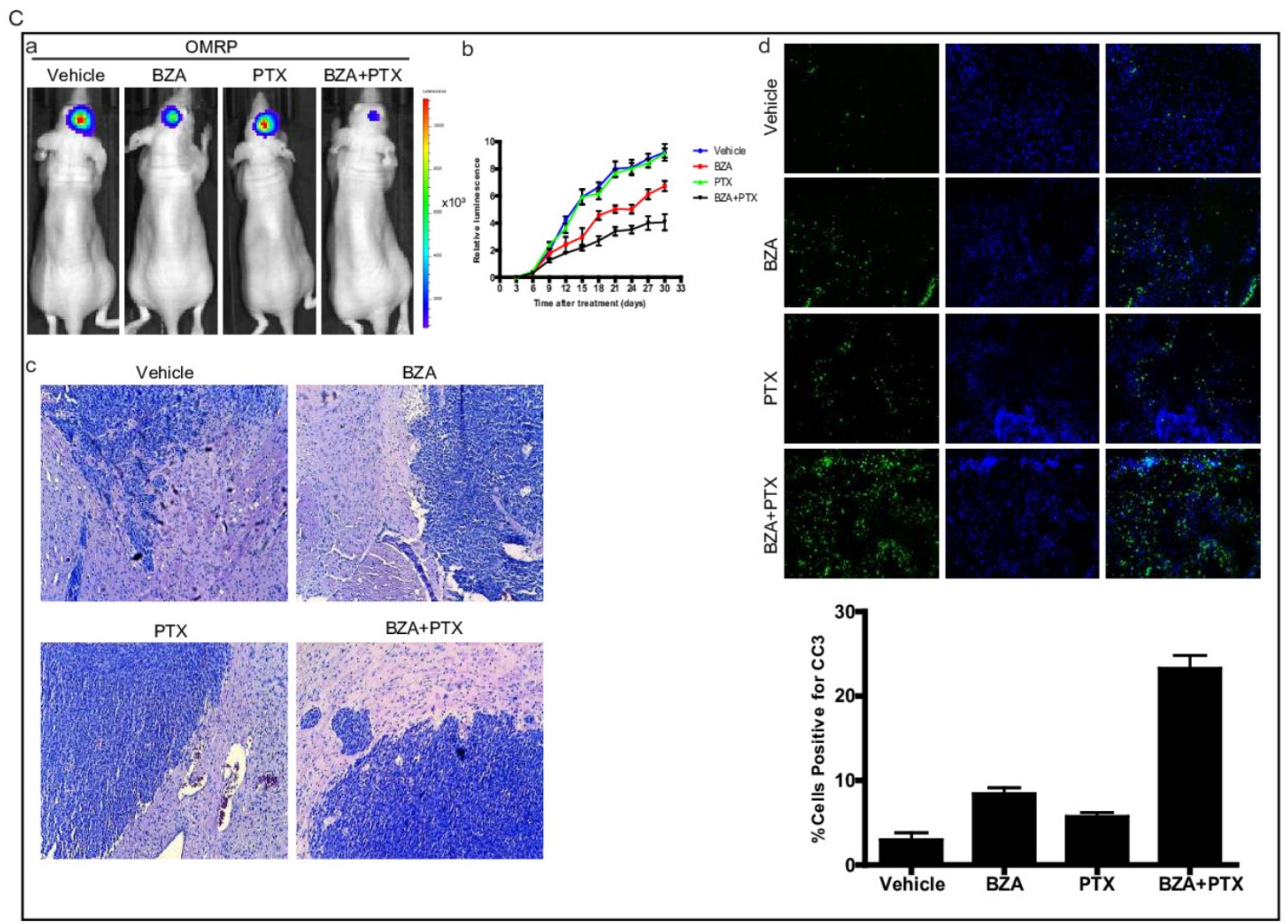

Figure 6. Bazedoxifene combined with paclitaxel further suppressed glioblastoma tumor growth in orthotopic GBM mouse model. (A). Luciferase U251MG cells based orthotopic GBM mouse model showed that combination of bazedoxifene (BZA, $5 \mathrm{mg} / \mathrm{kg}$ ) with paclitaxel (PTX, $10 \mathrm{mg} / \mathrm{kg}$ ) remarkably suppressed tumor growth. (a). Representative IVIS images. (b). Tumor growth rate measured by IVIS. (c). HE staining. (d). Western blotting detection of EMT related markers and YAP signaling. (e). immunofluorescence staining of cleaved caspase 3. (B). Luciferase D456 cells based orthotopic GBM mouse model further confirmed that combination of bazedoxifene with paclitaxel remarkably suppressed tumor growth. (a). Representative IVIS images. (b). Tumor growth rate measured by IVIS. (c). HE staining. (d). Western blotting detection of EMT related markers and YAP signaling. (e). immunofluorescence staining of cleaved caspase 3. (C). Luciferase OMRP cells based orthotopic GBM mouse model further confirmed that combination of bazedoxifene with paclitaxel remarkably suppressed tumor growth. (a). Representative IVIS images. (b). Tumor growth rate measured by IVIS. (c). HE staining. (d). immunofluorescence staining of cleaved caspase 3 . $*_{\mathrm{p}}<0.1 ; * * \mathrm{p}<0.01 ; *^{* *} \mathrm{p}<0.001$

The inhibitory effect of bazedoxifene on IL-6/GP130 signaling has been approved in several cancers including triple negative breast cancer, pancreatic cancer and head/neck cancer [5, 19, 23]. Previous works showed that bazedoxifene bonded to GP130 D1 domain through the hot spots Ile83, Phe36, Tyr94, and Asn92, which blocked the entry of IL-6 to GP130 and leaded to the inhibition of downstream signaling such as AKT, STAT3 and ERK [19]. As significant role of IL-6/GP130 in glioblastoma has been recognized long time ago. Glioblastoma samples tended to express higher levels of IL-6 compared to those in healthy brains [4]. And IL-6 was a poor prognostic factor for glioblastoma patients [17]. Moreover, GP130 as well as its downstream STAT3 signaling was tightly associated with the stemness of glioblastoma stem like cells [20]: GP130 blocking antibody drastically stem-like properties in GSCs. All these data indicate that IL-6/GP130 signaling plays essential role in the initiation and progression glioblastoma and targeting this pathway may overcome glioblastoma development. As IL-6/GP130 inhibitor, bazedoxifene opens a new window for glioblastoma treatment.

The oncogenic role of YAP in Hippo pathway has been approved in glioma by many groups. YAP overexpression promoted glioma cell growth by altering Wnt/beta-catenin [21]. Another study also demonstrated that YAP promoted cell migration and invasion by up-regulating N-cadherin and twist [27]. Consistently, our data also displayed that bazedoxifene treatment accelerated YAP phosphorylation. However, the underlying mechanism by which bazedoxifene enhances YAP phosphorylation and leads its degradation is still unclear for us. We hypothesize that there is cross-talk between IL-6/GP130 and YAP. Therefore, deactivation of GP130 by bazedoxifene may accelerate YAP phosphorylation and degradation.

In summary, our data defines the anticancer 
activity of bazedoxifene either alone or in combination with paclitaxel in glioblastoma in vitro and in vivo and builds strong rational to develop bazedoxifene as another therapeutic drug for glioblastoma patients.

\section{Acknowledgements}

We gratefully thank financial support from Natural Science Foundation of Shandong Province (ZR2019MH067), the Taishan Scholars Program (NO. tsqn2016070) and Qingdao People's Livelihood Science and Technology (17-3-3-18-nsh).

\section{Authors' contributions}

Weiwei Fu and Peng Zhao performed the major experiments. Hong $\mathrm{Li}$ and Haiyang $\mathrm{Fu}$ constructed luciferase glioblastoma cell lines. Xuejun Liu and Yingchao Liu collected the data. Jie $\mathrm{Wu}$ conducted statistical analyses. Weiwei $\mathrm{Fu}$ drafted and conceptualized manuscript.

\section{Competing Interests}

The authors have declared that no competing interest exists.

\section{References}

1 Barone TA, Gorski JW, Greenberg SJ, Plunkett RJ. Estrogen increases survival in an orthotopic model of glioblastoma. Journal of neuro-oncology. 2009; 95: $37-48$.

2 Chambers RC, Leoni P, Blanc-Brude OP, Wembridge DE, Laurent GJ Thrombin is a potent inducer of connective tissue growth factor production via proteolytic activation of protease-activated receptor-1. The Journal of biological chemistry. 2000; 275: 35584-35591.

3 Chen X, Wei J, Li C, Pierson CR, Finlay JL, Lin J. Blocking interleukin-6 signaling inhibits cell viability/proliferation, glycolysis, and colony forming activity of human medulloblastoma cells. International journal of oncology. 2018; 52: 571-578.

4 Choi C, Gillespie GY, Van Wagoner NJ, Benveniste EN. Fas engagement increases expression of interleukin- 6 in human glioma cells. Journal of neuro-oncology. 2002; 56: 13-19.

5 Choudhary MM, France TJ, Teknos TN, Kumar P. Interleukin-6 role in head and neck squamous cell carcinoma progression. World journal of otorhinolaryngology - head and neck surgery. 2016; 2: 90-97.

6 Fu S, Chen X, Lo HW, Lin J. Combined bazedoxifene and paclitaxel treatments inhibit cell viability, cell migration, colony formation, and tumor growth and induce apoptosis in breast cancer. Cancer letters. 2019; 448: 11-19.

$7 \mathrm{Fu} \mathrm{V}$, Plouffe SW, Guan KL. The Hippo pathway in organ development, homeostasis, and regeneration. Current opinion in cell biology. 2017; 49: 99-107.

8 Ishihara E, Nishina H. The Hippo-YAP Pathway Regulates 3D Organ Formation and Homeostasis. Cancers. 2018; 10

9 Kabat GC, Etgen AM, Rohan TE. Do steroid hormones play a role in the etiology of glioma? Cancer epidemiology, biomarkers \& prevention : a publication of the American Association for Cancer Research, cosponsored by the American Society of Preventive Oncology. 2010; 19: 2421-2427.

10 Komm BS, Chines AA. Bazedoxifene: the evolving role of third-generation selective estrogen-receptor modulators in the management of postmenopausal osteoporosis. Therapeutic advances in musculoskeletal disease. 2012; 4: 21-34.

11 Lan YL, Zou S, Wang X, Lou JC, Xing JS, Yu M et al. Update on the therapeutic significance of estrogen receptor beta in malignant gliomas. Oncotarget. 2017; 8: 81686-81696.

12 Ohgaki H, Kleihues P. Epidemiology and etiology of gliomas. Acta neuropathologica. 2005; 109: 93-108

13 Oike T, Suzuki Y, Sugawara K, Shirai K, Noda SE, Tamaki T et al. Radiotherapy plus concomitant adjuvant temozolomide for glioblastoma: Japanese mono-institutional results. PloS one. 2013; 8: e78943.

14 Overholtzer M, Zhang J, Smolen GA, Muir B, Li W, Sgroi DC et al. Transforming properties of YAP, a candidate oncogene on the chromosome 11q22 amplicon. Proceedings of the National Academy of Sciences of the United States of America. 2006; 103: 12405-12410.
15 Stupp R, Mason WP, van den Bent MJ, Weller M, Fisher B, Taphoorn MJ et al. Radiotherapy plus concomitant and adjuvant temozolomide for glioblastoma. The New England journal of medicine. 2005; 352: 987-996.

16 Stupp R, Hegi ME, Mason WP, van den Bent MJ, Taphoorn MJ, Janzer RC et al. Effects of radiotherapy with concomitant and adjuvant temozolomide versus radiotherapy alone on survival in glioblastoma in a randomised phase III study: 5-year analysis of the EORTC-NCIC trial. The Lancet Oncology. 2009; 10: 459-466.

17 Tchirkov A, Khalil T, Chautard E, Mokhtari K, Veronese L, Irthum B et al. Interleukin-6 gene amplification and shortened survival in glioblastoma patients. British journal of cancer. 2007; 96: 474-476.

18 Thakkar JP, Dolecek TA, Horbinski C, Ostrom QT, Lightner DD, Barnholtz-Sloan JS et al. Epidemiologic and molecular prognostic review of glioblastoma. Cancer epidemiology, biomarkers \& prevention : a publication of the American Association for Cancer Research, cosponsored by the American Society of Preventive Oncology. 2014; 23: 1985-1996.

19 Tian J, Chen X, Fu S, Zhang R, Pan L, Cao Y et al. Bazedoxifene is a novel IL-6/GP130 inhibitor for treating triple-negative breast cancer. Breast cancer research and treatment. 2019; 175: 553-566.

20 Wang $\mathrm{H}$, Lathia JD, Wu Q, Wang J, Li Z, Heddleston JM et al. Targeting interleukin 6 signaling suppresses glioma stem cell survival and tumor growth. Stem cells. 2009; 27: 2393-2404.

21 Wang Y, Pan P, Wang Z, Zhang Y, Xie P, Geng D et al. beta-catenin-mediated YAP signaling promotes human glioma growth. Journal of experimental \& clinical cancer research : CR. 2017; 36: 136.

22 Wilson TA, Karajannis MA, Harter DH. Glioblastoma multiforme: State of the art and future therapeutics. Surgical neurology international. 2014; 5: 64 .

23 Wu X, Cao Y, Xiao H, Li C, Lin J. Bazedoxifene as a Novel GP130 Inhibitor for Pancreatic Cancer Therapy. Molecular cancer therapeutics. 2016; 15: 2609-2619.

24 Yuan WC, Pepe-Mooney B, Galli GG, Dill MT, Huang HT, Hao M et al. NUAK2 is a critical YAP target in liver cancer. Nature communications. 2018; 9: 4834

25 Zanconato F, Cordenonsi M, Piccolo S. YAP/TAZ at the Roots of Cancer. Cancer cell. 2016; 29: 783-803.

26 Zhang J, Smolen GA, Haber DA. Negative regulation of YAP by LATS1 underscores evolutionary conservation of the Drosophila Hippo pathway. Cancer research. 2008; 68: 2789-2794.

27 Zhang $\mathrm{Y}$, Xie $\mathrm{P}$, Wang $\mathrm{X}$, Pan $\mathrm{P}$, Wang $\mathrm{Y}$, Zhang $\mathrm{H}$ et al. YAP Promotes Migration and Invasion of Human Glioma Cells. Journal of molecular neuroscience : MN. 2018; 64: 262-272.

28 Zhao B, Li L, Tumaneng $\mathrm{K}$, Wang $\mathrm{CY}$, Guan KL, A coordinated phosphorylation by Lats and $\mathrm{CK} 1$ regulates YAP stability through SCF(beta-TRCP). Genes \& development. 2010; 24: 72-85. 